University of Nebraska - Lincoln

DigitalCommons@University of Nebraska - Lincoln

Timothy J. Gay Publications

Research Papers in Physics and Astronomy

1996

\title{
Failure to observe electron circular dichroism in camphor
}

K. W. Trantham

The Australian National University, Canberra

M. E. Johnston

University of Saint Thomas, St. Paul, MN

Timothy J. Gay

University of Nebraska - Lincoln, tgay1@unl.edu

Follow this and additional works at: https://digitalcommons.unl.edu/physicsgay

Part of the Physics Commons

Trantham, K. W.; Johnston, M. E.; and Gay, Timothy J., "Failure to observe electron circular dichroism in camphor" (1996). Timothy J. Gay Publications. 56.

https://digitalcommons.unl.edu/physicsgay/56

This Article is brought to you for free and open access by the Research Papers in Physics and Astronomy at DigitalCommons@University of Nebraska - Lincoln. It has been accepted for inclusion in Timothy J. Gay Publications by an authorized administrator of DigitalCommons@University of Nebraska - Lincoln. 


\title{
Failure to observe electron circular dichroism in camphor
}

\author{
K.W. Trantham, M.E. Johnston, and T.J. Gay
}

\begin{abstract}
Analogous to optical circular dichroism, it is possible to have electron circular dichroism, the preferential transmission of longitudinally polarized electrons through a chiral medium. Using stereoisomers of camphor vapor as a scattering target, we looked for this effect at incident electron energies of $1,3,5,7$, or $10 \mathrm{eV}$. Within our detection limit $(\sim 0.02 \%)$ we have not observed a spin-dependent asymmetric transmission.
\end{abstract}

\begin{abstract}
Résumé : En analogie avec le dichrö̈sme circulaire optique, il est possible d'avoir un dichroïsme circulaire électronique, la propagation préférentielle d'électrons polarisés longitudinalement à travers un milieu chiral. Utilisant de la vapeur de stéréoisomères de camphre comme cible, nous avons cherché cet effet avec des électrons d'énergie incidente de 1, 3, 5, 7 et $10 \mathrm{eV}$. À la précision de nos appareils de détection $(\sim 0.02 \%)$ nous n'avons pas observé d'effet dépendant du spin dans la transmission.
\end{abstract}

[Traduit par la rédaction]

Optical activity is the rotation of the plane of polarization of linearly polarized light as it passes through a chiral medium. This phenomenon was first observed by Arago in quartz and by Biot in liquids. Circular dichroism, the preferential absorption of left- or right-handed circularity polarized light in a chiral medium, is a related phenomenon. From symmetry arguments, it can be shown that similar phenomena can occur with electron scattering from chiral targets. First proposed by Farago [1, 2], electron optical activity is the rotation of the transverse spin vector in a plane perpendicular to the momentum as a beam of electrons traverse a chiral medium, whereas electron circular dichroism is the preferential absorption of left- or right-handed longitudinally polarized electrons.

As a consequence of electron circular dichroism, an initially unpolarized electron beam can develop a polarization component in the plane of scattering. Beerlage et al. [3] first searched for this effect but failed to see it within their experimental limits. Another experimental search for electron circular dichroism by Campbell and Farago [4] looked for preferential transmission of longitudinally polarized electrons through stereoisomers of camphor. They measured the asymmetry

$\mathscr{A} \equiv \frac{\left(I_{\uparrow}-I_{\downarrow}\right)}{\left(I_{\uparrow}+I_{\downarrow}\right)}$

Received August 5, 1995. Accepted March 15, 1996.

K.W. Trantham, ${ }^{1}$ M.E. Johnston, ${ }^{2}$ and T.J. Gay. ${ }^{3}$ Behlen Laboratory of Physics, University of Nebraska, Linclon, NE 68588-0111, U.S.A.

1 Present address: Electron Physics Group, Research School of Physical Sciences, The Australian National University, Canberra, ACT 0200, Australia.

2 Department of Physics, University of Saint Thomas, 2115

Summit Avenue, Saint Paul, MN 55105-1096, U.S.A.

3 The author to whom all correspondence should be addressed. Phone: (402) 472-2786; FAX: (402) 472-2879; e-mail: tgay@unlinfo.unl.edu. where $I_{\uparrow}(\downarrow)$ is the transmitted intensity of electrons for a given incident current with spins aligned (antialigned) with their momenta. Note that $\mathscr{A}$ is required by symmetry to change sign when the target handedness is reversed, and will depend linearly on the incident electron polarization. Campbell and Farago found $\mathscr{A}$ to be $23(11) \times 10^{-4}$ for $d$-camphor and $-50(17) \times 10^{-4}$ for its enantiomorph ( $l$-camphor) at an incident electron energy of $5 \mathrm{eV}$ and polarization of 0.28 .

These results are intriguing, since they give the first evidence of electron circular dichroism. However, the measured magnitude of $\mathscr{A}$ is much larger than that expected from present dynamical models. Proposed by Kessler [5], one mechanism involves plural scattering of the incident electron within the framework of the molecule. Here, the electron is first transversely polarized by spin-orbit interactions (Mott scattering), and a final collision within the molecule projects some component of polarization along the electron's momentum. This final collision acts in a manner analogous to an electrostatic spin rotator. Thus a nonzero longitudinal polarization develops and does not average to zero over all orientations of the chiral target. Kessler estimated the largest polarization for a carbon chiral center to be $\sim 10^{-4}$. In a second paper, Hayashi [6] obtained $10^{-6}$ for similar scattering conditions. One would then expect the transmission asymmetry $\mathscr{A}$ be of the same order.

Another approach is similar to the conventional optical model describing ordinary optical activity. Essentially, the incident electron induces both an electric $(e)$ and magnetic (m) dipole moment in the target. Although an electric dipole moment can be induced in any molecule, the magnetic dipole moment arises only in chiral molecules from lack of inversion symmetry. Since induced moments depend only on the incident electron momentum, the interaction between the electron and these moments $\left(H^{\prime} \propto \hat{p} ; \hat{\sigma}\right)$ will depend on the spin direction. The relevant parameter characterizing the strength of this interaction is the polarizability of the target molecule. The strength of this interaction can be estimated from camphor's optical rotary power and other optical properties. Using this approach, Walker [7] inferred $\mathscr{A}$ to be $\sim 10^{-23}$. 
Recently Gallup ${ }^{4}$ has calculated ab initio the strength of this interaction and finds $\mathscr{A} \sim 10^{-4}$.

Yet another theoretical formalism applied to this problem is the bound helical electron model. As originally shown $[8,9]$, the chiral molecule develops a net "helicity density," which means that molecular orbital spin and momentum are correlated. Spin-dependent scattering from such a target arises due to a velocity-dependent exchange amplitude. From this model, Hegstrom obtained $\mathscr{A} \cong \eta(\alpha Z)^{2}$, where $\alpha$ is the fine structure constant, and $Z$ is the charge of the nucleus at the chiral center of the molecule. The parameter $\eta$ accounts for the degree of molecular chirality, and is typically about $10^{-2}$. Thus, for most carbon-based chiral molecules such as camphor, $\mathscr{A} \sim 10^{-5}$.

Since the results of Campbell and Farago [4] are inconsistent with a general theoretical result of $10^{-4}-10^{-5}$, its origin is unclear. To gain insight into this problem, we repeated their experiment. As did Campbell and Farago, we measured $\mathscr{A}$ with an incident electron energy of $5 \mathrm{eV}$ and used stereoisomers of camphor as a scattering target. Additionally, at the suggestion of Burrow ${ }^{5}$ (see also ref. 10), $A$ was measured at $1 \mathrm{eV}$, where a negative ion resonance is known to exist. By lengthening the collision time, one might expect that the incident electron and target molecule could "sample each other's handedness," thus enhancing $\mathscr{A}$. To supplement this investigation, $A$ was also measured at 3,7 , and $10 \mathrm{eV}$.

Our apparatus was designed to be as axially symmetric as possible to reduce possible instrumental asymmetries. It is composed of three main components: a polarized electron source (1 in Fig. 1), an electron polarimeter (4 in Fig. 1), and a camphor target chamber (6 in Fig. 1). Essentially all electromagnetic fields are axially symmetric about the beam axis, with the exception of small steering fields.

The polarized electron source is based on photoemission from GaAs. The electrons are extracted and transported longitudinally polarized with cylindrical electron optics (3 in Fig. 1). The photoemission laser light ( 2 in Fig. 1) enters the apparatus almost collinear with the electron-beam axis. The light undergoes a reflection from a polished stainless steel electron aperture in the beam line and illuminates the photoemitter at near-normal incidence. Thus, the electrons are almost completely longitudinally polarized. The operation of this type of electron source is similar to that discussed in Pierce et al. [11]. The electron helicity is controlled via the laser's circular polarization with a liquid crystal variable retarder, available from Meadowlark optics (LVR-200).

The electron polarization is measured optically. The polarimeter is based on exchange excitation of an atomic target by the polarized electrons and measuring the circular polarization (relative Stokes parameter $S / I$ ) of the subsequent optical decay. We used the $3 p^{3} D_{3} \rightarrow 3 s^{3} P_{2}(6204 \AA(1 \AA=$ $\left.10^{-10} \mathrm{~m}\right)$ ) transition in $\mathrm{Ne}$, and observed the decay at a polar angle of $135^{\circ}$ from the beam axis. Under these conditions, the electron polarization $\left(P_{\mathrm{e}}\right)$ is found from

$\frac{S}{I}=\frac{\sqrt{2}}{3}\left(1-\frac{17}{9} \frac{M}{I}\right) P_{\mathrm{e}}$

where $M / I$ is the linear polarization of the fluorescence mea-

4 G.A. Gallup, private communication.

5 P.D. Burrow, private communication.
Fig. 1. Apparatus used to investigate electron circular dichroism in camphor. Component indices 1-10 are described in the text.

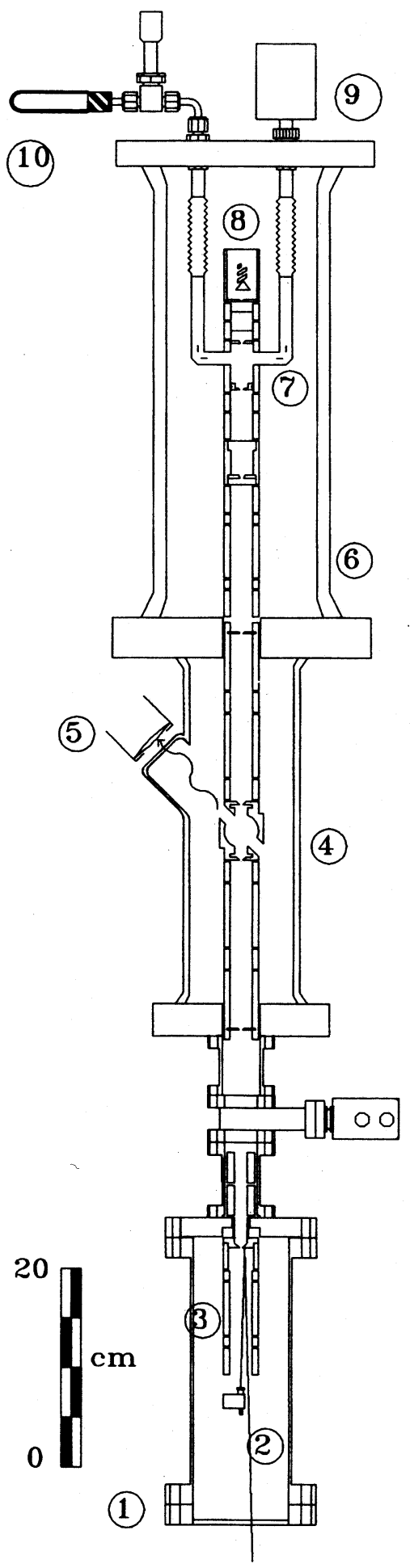

sured at $0^{\circ}$ and $90^{\circ}$ with respect to the beam axis. Only the first lens of the optical polarimeter (5 in Fig. 1) is shown in Fig. 1. More detailed discussions of optical electron polarimetry can be found in refs. 12 and 13. From these measurements, the electron polarization was found to vary between $27 \%$ for bulk GaAs and $45 \%$ for thin, epitaxially grown crystals. 
Fig. 2. Typical retarding field spectrum used to determine proper potential settings to accept only elastically scattered electrons. The broken line indicates threshold of transmission; the arrow indicates the proper potential setting.

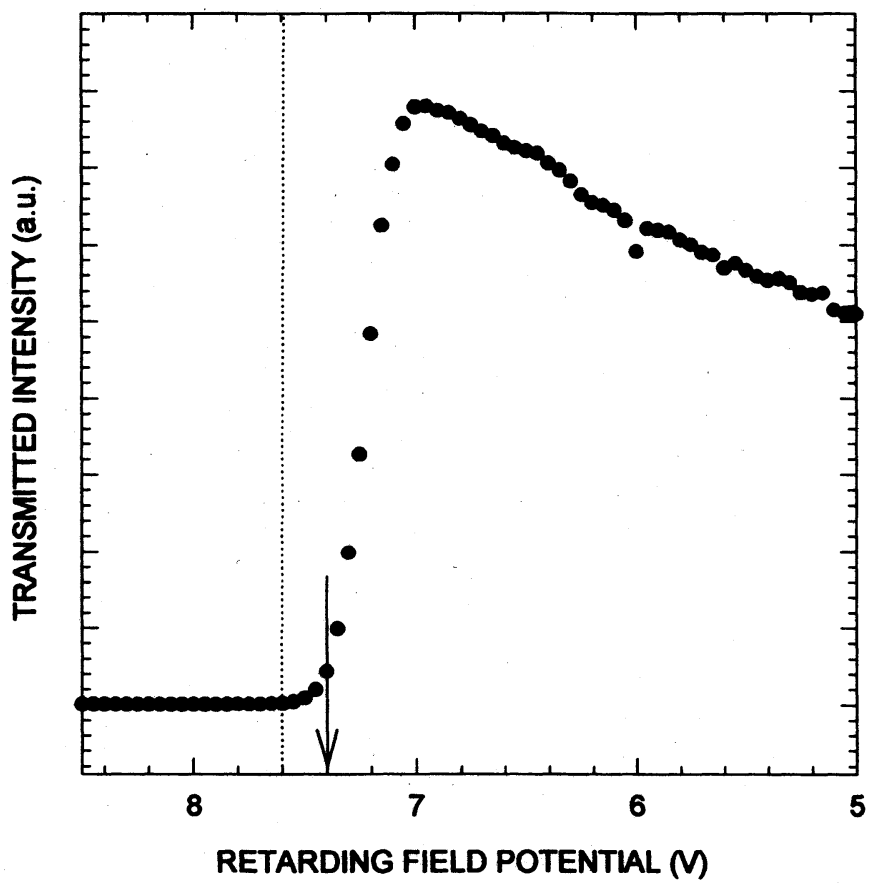

In the target chamber, the electron beam was transported from the polarimeter and focused into a vapor target cell ( 7 in Fig. 1). Following the target was a retarding field energy analyzer (RFA) and an electron multiplier (8 in Fig. 1) for single-particle detection of the transmitted electrons. We used both continuous dynode multipliers ("channeltrons") and discrete dynode multipliers for particle detection. The target cell had a port for the introduction of camphor and another for monitoring the target pressure with a capacitance manometer (9 in Fig. 1). The RFA uses a screen placed immediately after the target exit aperture. With its potential set properly, only those electrons which have suffered no more than a $200 \mathrm{meV}$ energy loss will be transmitted to the detector. We used the computer program SIMION [14] to determine that the geometry of the detector and target apertures allow only forward-scattered electrons within a half-angle of $2^{\circ}$ to be detected.

The correct RFA potential is found by decreasing its applied voltage to reject the electron beam and identifying the threshold of transmission (broken line in Fig. 2). The RFA is then set $200 \mathrm{mV}$ above this threshold as indicated by the arrow. The gas cell potential is set in a similar manner; for a given energy, $\mathcal{V} \mathrm{eV}$, its potential is set $\mathcal{V} \mathrm{V}$ above the lowest potential that transmits the electron beam.

Camphor vapor is supplied from a vial at room temperature (10 in Fig. 1) containing a solid sample. A target pressure of $\sim 1 \mathrm{mTorr}(1$ Torr $=133.32 \mathrm{~Pa})$ was required to attenuate the electron beam by a factor of 4 or 5 , the same conditions reported by Campbell and Farago [4]. With a vapor pressure of $\sim 200$ mTorr at room temperature, we experienced little difficulty in maintaining sufficient pressure in the target cell. However, the electron optics and target chamber needed to be warmed slightly $\left(\sim 40^{\circ} \mathrm{C}\right)$ with an infrared heat lamp to
Fig. 3. Transmission asymmetries, $\mathscr{A}$, compared with the results of Campbell and Farago [4] (C \& F). All data are normalized to electron polarization.

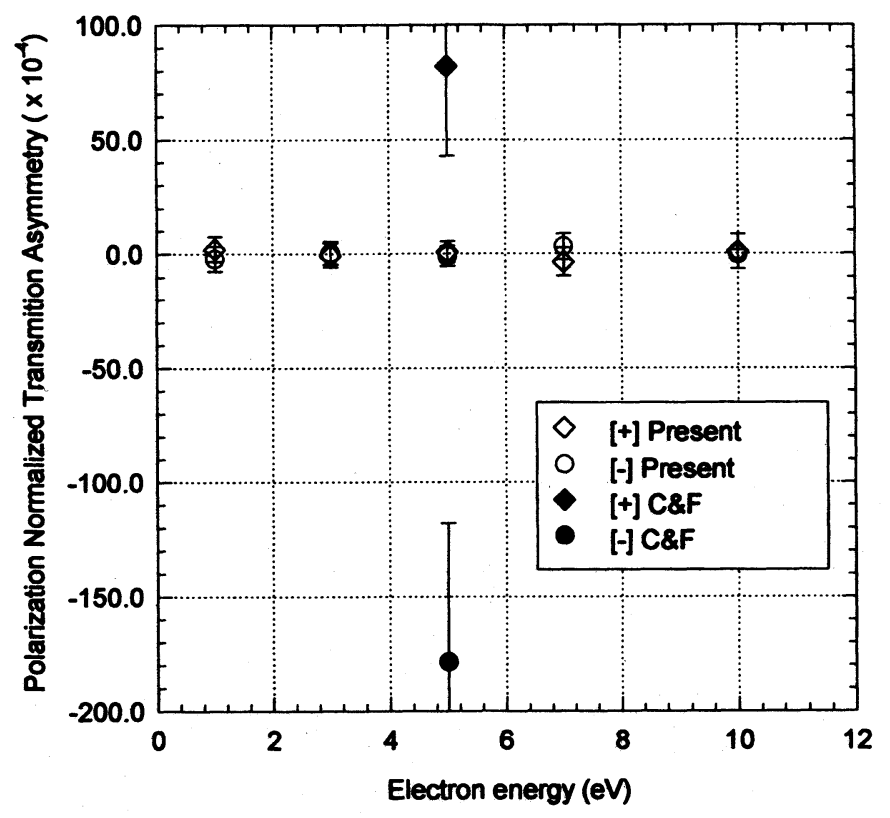

prevent anomalous tuning effects due to camphor "plating" of the transport optics. To ensure chiral purity of our camphor supply, the optical specific rotation was checked for each isomer by measuring the degree of rotation of plane polarized light with a known concentration of camphor dissolved in acetone (see, for example, ref. 15).

Total scattering cross sections $\left(\sigma_{\text {tot }}\right)$ were measured first to verify our ability to handle low-energy electrons and to control the flow of vapor into the target chamber. We confirmed qualitatively the existence of a negative ion resonance in the region of $1 \mathrm{eV}$, the effects of which were first observed by Stephen et al. [10].

Our asymmetry measurements are a culmination of many blocks of data taken over the course of several weeks. Each block contains $\sim 500$ individual asymmetry measurements, each obtained by toggling the electron's helicity and recording $I_{\uparrow}$ and $I_{\downarrow}$, as defined above. The temporal order of data acquisition $\left(I_{\uparrow}, I_{\downarrow}\right.$ or $\left.I_{\downarrow}, I_{\uparrow}\right)$ was randomly set for each asymmetry. For a given target handedness, $\sim 125$ asymmetries were taken, with $\sim 2 \mathrm{~s}$ of data accumulation time for each helicity setting. The target handedness was then changed and another $\sim 125$ asymmetries accumulated. Care was taken to insure that residual target molecules had been pumped out before a new target was admitted to the chamber. Target handedness was typically switched four times for a given block of data. A mean and standard error were then computed for each isomer from the $\sim 250$ available asymmetries, yielding an $\mathscr{A}_{d}^{\text {raw }}$ and $\mathscr{A}_{l}^{\text {raw }}$ for a given block of data Since $\mathscr{A}_{d}^{\text {raw }}$ and $\mathscr{A}_{l}^{\text {raw }}$ should be equal in magnitude and opposite in sign, the instrumental asymmetries were eliminated by computing the final asymmetries using

$\mathscr{A}_{L}=\frac{\mathscr{A}_{l}^{\text {raw }}}{2}-\frac{\mathscr{A}_{d}^{\text {raw }}}{2}$
$\mathscr{A}_{D}=\frac{\mathscr{A}_{d}^{\text {raw }}}{2}-\frac{\mathscr{A}_{l}^{\text {raw }}}{2}$ 
The instrumental asymmetry

$\mathscr{A}_{I}=\frac{\mathscr{A}_{l}^{\text {raw }}}{2}+\frac{\mathscr{A}_{d}^{\text {raw }}}{2}$

was typically $2 \times 10^{-4}$, in agreement with asymmetry runs made with argon as the target gas. For each block of data at a given energy, $\mathscr{A}$ was normalized to the measured electron polarization. Figure 3 shows our results at energies of 1, 3, 5 , 7, and $10 \mathrm{eV}$, along with the results of Campbell and Farago [4], normalized to their polarization, for comparison. Although our results are constant with dynamical models sketched above, we have not been able to verify Campbell and Farago's results at $5 \mathrm{eV}$. Moreover, we were not able to observe an enhancement in $\mathscr{A}$ near the resonance at $1 \mathrm{eV}$.

It is unclear at present why our measurements are inconsistent with Campbell and Farago's [4] results. Recently, Mayer and Kessler [16] repeated this same experiment. They confirm our results with camphor, but also observed nonzero values of $\mathscr{A}$ with a chiral molecule containing ytterbium. Farago's [1, 2] original ideas of electron optical activity and circular dichroism represent basic physical phenomena; they are worth trying to observe and understand. Therefore, we intend to look for both of these effects in other materials with higher values of $Z$, such as 3-bromocamphor. It may be possible to measure asymmetries faster using a phase-sensitive detection scheme. This will be accomplished by sinusoidally modulating the electron polarization and measuring the transmitted current with a lock-in amplifier.

\section{Acknowledgments}

This work was supported by National Science Foundation grant PHY-9504350. The authors wish to acknowledge numerous useful conversations with G.A. Gallup and Gregory A. Mulhollan of the Stanford Linear Accelerator Center for providing our thin, epitaxially grown GaAs crystals.

\section{References}

1. P.S. Farago. J. Phys. B: At. Mol. Phys. 13, L567 (1980).

2. P.S. Farago. J. Phys. B: At. Mol. Phys. 14, L743 (1981).

3. M.J. M. Beerlage, P.S. Farago, and M.J. Van der Wiel. J. Phys. B: At. Mol. Phys. 14, 3245 (1981).

4. D.M. Campbell and P.S. Farago. J. Phys. B: At. Mol. Phys. 20, 5133 (1987).

5. J. Kessler. J. Phys. B: At. Mol. Phys. 15, L101 (1982).

6. S. Hyashi. J. Phys. B: At. Mol. Phys. 18, 1229 (1985).

7. D.W. Walker. J. Phys. B: At. Mol. Phys. 15, L289 (1982).

8. R.A. Hegstrom. Nature (London), 297, 643 (1982).

9. A. Rich, J. Van House, and R.A. Hegstrom. Phys. Rev. Lett. 48, 1341 (1982).

10. T.M. Stephen, X. Shi, and P.D. Burrow. J. Phys. B: At. Mol. Opt. Phys. 21, L169 (1988).

11. D.T. Pierce, R.J. Celotta, G.C. Wang, W.N. Unertl, A. Galejs, C.E. Kuyatt, and S.R. Mielczarek. Rev. Sci. Instrum. 51, 478 (1980).

12. J.E. Furst, W.M.K.P. Wijayaratna, D.H. Madison, and T.J. Gay. Phys. Rev. A: At. Mol. Opt. Phys. 47, 3775 (1993).

13. T.J. Gay, J.E. Furst, K.W. Trantham, and W.M.K.P. Wijayaratna. Phys. Rev. A: At. Mol. Opt. Phys. 53, 1623 (1995).

14. SIMION (1988), available from D.A. Dahl, Idaho National Engineering Laboratory.

15. L.D. Barron. Molecular light scattering and optical activity. Cambridge University Press, New York. 1982.

16. S. Mayer and J. Kessler. Phys. Rev. Lett. 74, 4803 (1995). 